Research Article

\title{
Serum Levels of lncRNA CCHE1 and TCF21 in Patients with Coronary Artery Disease and Their Clinical Significances
}

\author{
Miaomiao Liu $(\mathbb{D}$, Ying Zhang, Xiantong Cao, and Xue Wang \\ Department of Cardiovascular Surgery, First Affiliated Hospital of Xi'an Jiaotong University, Xi'an, China \\ Correspondence should be addressed to Miaomiao Liu; maggielmm@xjtufh.edu.cn
}

Received 27 October 2021; Accepted 1 December 2021; Published 21 December 2021

Academic Editor: Francesco Busard?

Copyright ( 2021 Miaomiao Liu et al. This is an open access article distributed under the Creative Commons Attribution License, which permits unrestricted use, distribution, and reproduction in any medium, provided the original work is properly cited.

\begin{abstract}
Objective. To detect serum level changes of CCHE1 and TCF21 in coronary artery disease (CAD) patients and to explore their clinical significances. Patients and Methods. A total of 150 CAD patients were divided into the mild lesion group ( $n=52$ ), moderate lesion group $(n=48)$, and severe lesion group $(n=50)$, respectively, according to the Gensini score. In addition, they were divided into single vessel lesion $(n=42)$, two vessel lesions $(n=49)$, and three vessel lesions group $(n=59)$, respectively. Serum levels of CCHE1 and TCF21 in CAD patients were detected by quantitative real-time polymerase chain reaction (qRTPCR). Spearman's rank correlation was conducted to assess the relationship between levels of CCHE1 and TCF21 and severity and numbers of vessel lesions in CAD. Pearson's correlation test was used for analyzing the correlation between CCHE1 and TCF21 levels. A multivariable logistic regression test was performed to evaluate the influences of CCHE1 and TCF21 levels on CAD severity and the occurrence of cardiovascular events within 3 years of follow-up. Results. Significant differences in incidences of diabetes and hypertension were identified in CAD patients divided according to CAD severity. In addition, significant differences in incidences of drinking, diabetes, and hypertension were identified in CAD patients divided according to numbers of vessel lesions. The serum level of CCHE1 was positively related to CAD severity and numbers of vessel lesions, while TCF21 displayed a negative relationship. During the 3-year follow-up, the incidence of cardiovascular events was $39.3 \%$ (59/150). CAD severity, numbers of vessel lesions, and serum levels of CCHE1 and TCF21 were independent factors influencing the occurrence of cardiovascular events in CAD patients. Conclusions. The increased serum level of CCHE1 and decreased TCF21 level are closely related to CAD severity, which are able to influence the prognosis in CAD patients.
\end{abstract}

\section{Introduction}

Coronary artery disease $(\mathrm{CAD})$ is one of the major reasons for global death [1]. CAD is mainly caused by atherosclerosis [2]. Phenotypes of vascular smooth muscle cells (VSMCs) are changed following injuries, and they migrate to intima and aggregate ECM. VSMCs are of significance in the development of atherosclerosis [3].

Long non-coding RNAs (lncRNAs) contain more than 200 nucleotides in length, and they are unable to encode proteins [4]. Abnormally expressed IncRNAs have been detected in the heart or circulating system of myocardial infarction (MI) patients $[5,6]$. A latest study has uncovered that lncRNA LIPCAR is capable of monitoring heart failure at post-MI, serving as a promising biomarker [7]. Further, MIAT controls advanced atherosclerotic lesion formation and plaque destabilization [8]. The SNHG1-driven selfreinforcing regulatory network promoted cardiac regeneration and repair after myocardial infarction [9]. IncRNA CCHE1 (cervical carcinoma high expressed 1) has been recently discovered [10]. Microarray analysis showed upregulated CCHE1 in breast cancer profiling, suggesting that CCHE1 may have a certain impact on breast cancer progression $[11,12]$. The correlation between CCHE1 and CAD, however, is rarely reported.

TCF21 locates on 6q23-q24, which was discovered in the research on the location of chromosomal heterozygosity in 2006 by Smith et al. [13]. TCF21 is a metastasis-suppressor gene. It is reported that TCF21 is extensively expressed in stromal cells or derived cells during the development of cardiovascular system, urogenital system, and respiratory system [14]. It largely affects cell growth and differentiation. 
A previous study has proposed that LINC00163 alleviates lung cancer progression by upregulating TCF21 [15]. In this paper, we mainly explored the clinical significances of CCHE1 and TCF21 in CAD patients. Our findings can provide novel ideas in clinical treatment of CAD.

\section{Patients and Methods}

2.1. Baseline Characteristics. A total of 150 patients admitted in our hospital from June 2017 to May 2019 because of chest tightness, chest pain, or acute MI were enrolled. All patients underwent coronary angiography and treated according to the "2012 ACCF/AHA Focused Update of the Guideline for the Management of Patients with Unstable Angina/ non-ST-Elevation Myocardial Infarction" [16]. Gensini score was graded depending on the stenosis and the locations of vessel narrowing ( 1 grade: stenosis < 25\%, 2 grades: 26\%-50\% stenosis, 4 grades: $51-75 \%$ stenosis, 8 grades: $76 \%-$ 90\%, 16 grades: $90 \%-98 \%$, and 32 grades: complete stenosis) [17]. Then, the corresponding coefficients were determined by the location of different narrowing branches of coronary artery (left coronary artery: $\times 5$, proximal LAD: $\times 2.5$, midLAD: $\times 1.5$, and distal LAD: $\times 1$ ). We divided CAD patients into the mild lesion group (1.0-26.1 grades, $n=52)$, the moderate lesion group (26.2-51.4 grades, $n=48)$, and the severe lesion group ( $\geq 51.5$ grades, $n=50$ ) according to the Gensini score. In addition, patients were classified by the number of vessel lesions to the single-vessel lesion group: $\geq 50 \%$ of stenosis of any single vessel in LAD, left circumflex, and right coronary artery; the two-vessel lesion group: $\geq 50 \%$ of stenosis of any two vessels in LAD, left circumflex and right coronary artery; three vessel lesions group: stenosis in LAD, left circumflex and right coronary artery, or lesions in left main coronary artery combined with the right coronary artery. Patients with malignant tumors, liver or kidney insufficiency, acute/chronic infection, pregnant or lactating women, or those unwilling to participate in were excluded. The study was reviewed and approved by the Ethics Committee of First Affiliated Hospital of Xi'an Jiaotong University. All patients signed the informed consent.

2.2. Laboratory Examinations. Serum levels of TC (total cholesterol), TG (triglyceride), LDL-C (low-density lipoprotein cholesterol), and HDL-C (high-density lipoprotein cholesterol) were determined using the automatic biochemical instrument. Hcy (homocysteine) and CRP (C-reactive protein) were detected by enzyme-linked immunosorbent assay (ELISA) (R\&D Systems, Minneapolis, MN, USA). LVEF (left ventricular ejection fraction) was recorded by echocardiography.

2.3. Quantitative Real-Time Polymerase Chain Reaction ( $q R T-P C R)$. Serum RNA was isolated using TRIzol and quantified by NanoDrop 2000 (Thermo Fisher Scientific, Waltham, MA, USA). RNA was reversely transcribed to complementary deoxyribose nucleic acid (cDNA) with the PrimeScript $^{\mathrm{TM}}$ RT Master Mix and amplified by TBGreen ${ }^{\circledR}$ Premix Ex $\mathrm{Taq}^{\mathrm{TM}}$ II (TaKaRa, Tokyo, Japan). Thermal cycle reactions were as follows: $30 \mathrm{~s}$ at $95^{\circ} \mathrm{C}$ and 40 cycles for $5 \mathrm{~s}$ at $95^{\circ} \mathrm{C}$ and $30 \mathrm{~s}$ at $60^{\circ} \mathrm{C}$. Primer sequences were as follows: CCHE $1-5^{\prime}$-AAGGTCCCAGGATACTCGC-3' (forward) and $5^{\prime}$-GTGTCGTGGACTGGCAAAAT- $3^{\prime}$ (reverse); TCF2 $1-5^{\prime}$-CCAGCTACATCGCCCACTTG-3' (forward) and $5^{\prime}$-CTTTCAGGTCACTCTCGGGTTTC-3' (reverse); and GAPDH $-5^{\prime}$-ACCACAGTCCATGCCATCAC-3' (forward) and $5^{\prime}$-TCCACCACCCTGTTGCTGTA-3' (reverse).

2.4. Follow-Up. Patients were followed up for three years by review, telephone, or e-mail. Cardiovascular events were recorded, including angina pectoris, MI, arrhythmia, sudden cardiac death, and stroke.

2.5. Statistical Analyses. Data were processed by SPSS 20.0. Measurement data were expressed as the mean \pm SD and compared using a $t$-test or variance analysis. Counting data were expressed as composition ratio or rate (\%) and compared by a chi-square test. Spearman's rank correlation was conducted to assess the relationship between levels of CCHE1 and TCF21 and severity and numbers of vessel lesions in CAD. Pearson's correlation test was used for analyzing the correlation between CCHE1 and TCF21 levels. A multivariable logistic regression test was performed to analyze the influences of CCHE1 and TCF21 levels on CAD severity and the occurrence of cardiovascular events within 3 years of follow-up. $p<0.05$ was statistically significant.

\section{Results}

3.1. Clinical Data of CAD Patients. Significant differences in incidences of diabetes and hypertension were identified in CAD patients in the mild lesion group $(n=52)$, the moderate lesion group $(n=48)$, and the severe lesion group $(n=50$ ) divided according to the Gensini score. Besides, significant differences in incidences of drinking, diabetes and hypertension were identified in CAD patients in the single-vessel lesion $(n=42)$, two-vessel lesion $(n=49)$, and three-vessel lesion groups $(n=59)$ (Tables 1 and 2$)$. It is indicated that diabetes and hypertension could affect CAD severity, while diabetes, hypertension, and drinking could affect numbers of vessel lesions.

3.2. Serum Levels of CCHE1 and TCF21 in CAD Patients with Different Severities. Serum levels of CCHE1 and TCF21 in CAD patients with different severities were detected by qRT-PCR. CCHE1 level increased with the aggravation of CAD, manifesting as the lowest level in the mild lesion group and the highest level in the severe lesion group (Figure 1(a)). On the contrary, TCF21 level decreased with the severity of CAD (Figure 1(b)).

3.3. Serum Levels of CCHE1 and TCF21 in CAD Patients with Different Numbers of Vessel Lesions. We thereafter detected CCHE1 and TCF21 levels in CAD patients classified by numbers of vessel lesions. The lowest level of CCHE1 was detected in the single-vessel lesion group, and the highest level was detected in the three-vessel lesion group (Figure 2(a)). TCF21 level gradually decreased with the increased numbers of vessel lesions (Figure 2(b)). 
TABLE 1: Clinical data of CAD patients with different severities.

\begin{tabular}{|c|c|c|c|c|}
\hline Variable & Mild lesion $(n=52)$ & Moderate lesion $(n=48)$ & Severe lesion $(n=50)$ & $p$ \\
\hline Male/female & $25 / 27$ & $24 / 24$ & $27 / 23$ & 0.831 \\
\hline Age & $56.23 \pm 7.85$ & $54.91 \pm 6.08$ & $55.21 \pm 5.45$ & 0.159 \\
\hline BMI $\left(\mathrm{kg} / \mathrm{m}^{2}\right)$ & $24.73 \pm 3.11$ & $23.01 \pm 3.65$ & $23.83 \pm 3.94$ & 0.073 \\
\hline Smoking & $20(38.46 \%)$ & $17(35.42 \%)$ & $23(46 \%)$ & 0.543 \\
\hline Drinking & $12(23.08 \%)$ & $14(29.17 \%)$ & $15(30 \%)$ & 0.693 \\
\hline TC $(\mathrm{mmol} / \mathrm{L})$ & $4.89 \pm 1.07$ & $4.08 \pm 1.33$ & $4.36 \pm 1.54$ & 0.615 \\
\hline $\mathrm{TG}(\mathrm{mmol} / \mathrm{L})$ & $1.64 \pm 0.46$ & $1.73 \pm 0.55$ & $1.74 \pm 0.54$ & 0.368 \\
\hline LDL-C (mmol/L) & $1.44 \pm 0.62$ & $1.38 \pm 0.56$ & $1.43 \pm 0.71$ & 0.741 \\
\hline HDL-C (mmol/L) & $2.56 \pm 1.72$ & $2.65 \pm 1.48$ & $2.71 \pm 1.82$ & 0.587 \\
\hline Hcy $(\mathrm{mmol} / \mathrm{L})$ & $14.46 \pm 2.88$ & $15.84 \pm 3.06$ & $15.38 \pm 2.89$ & 0.087 \\
\hline CRP (mg/L) & $12.27 \pm 5.23$ & $13.61 \pm 5.66$ & $13.84 \pm 5.68$ & 0.103 \\
\hline LVEF (\%) & $64.13 \pm 10.22$ & $62.33 \pm 9.36$ & $60.87 \pm 8.91$ & 0.067 \\
\hline Diabetes (\%) & $5(9.62 \%)$ & $4(8.33 \%)$ & $13(26 \%)$ & 0.021 \\
\hline Hypertension (\%) & $4(7.69 \%)$ & $7(14.58 \%)$ & $18(16 \%)$ & 0.001 \\
\hline
\end{tabular}

BMI: body mass index; TC: total cholesterol; TG: triglyceride; LDL-C: low-density lipoprotein cholesterol; HDL-C: high-density lipoprotein cholesterol; Hcy: homocysteine; CRP: C-reactive protein; LVEF: left ventricular ejection fraction.

TABLE 2: Clinical data of CAD patients with different numbers of vessel lesions.

\begin{tabular}{|c|c|c|c|c|}
\hline Variable & Single vessel $(n=42)$ & Two vessels $(n=49)$ & Three vessels $(n=59)$ & $p$ \\
\hline Male/female & $23 / 19$ & $22 / 27$ & $31 / 28$ & 0.601 \\
\hline Age & $57.27 \pm 6.95$ & $55.68 \pm 5.18$ & $57.11 \pm 6.13$ & 0.338 \\
\hline BMI $\left(\mathrm{kg} / \mathrm{m}^{2}\right)$ & $22.13 \pm 4.03$ & $23.32 \pm 4.53$ & $23.12 \pm 3.83$ & 0.275 \\
\hline Smoking & $17(40.48 \%)$ & $19(38.78 \%)$ & $24(40.68 \%)$ & 0.977 \\
\hline Drinking & $6(14.29 \%)$ & $10(20.41 \%)$ & $25(42.37 \%)$ & 0.003 \\
\hline TC $(\mathrm{mmol} / \mathrm{L})$ & $4.11 \pm 2.37$ & $4.41 \pm 2.54$ & $4.26 \pm 2.05$ & 0.652 \\
\hline $\mathrm{TG}(\mathrm{mmol} / \mathrm{L})$ & $1.77 \pm 0.73$ & $1.86 \pm 0.71$ & $1.92 \pm 0.83$ & 0.076 \\
\hline LDL-C (mmol/L) & $1.37 \pm 0.51$ & $1.48 \pm 0.56$ & $1.43 \pm 0.71$ & 0.198 \\
\hline HDL-C (mmol/L) & $2.56 \pm 1.72$ & $2.73 \pm 1.98$ & $2.82 \pm 2.13$ & 0.227 \\
\hline Hcy $(\mathrm{mmol} / \mathrm{L})$ & $13.86 \pm 3.18$ & $14.21 \pm 3.22$ & $14.88 \pm 3.89$ & 0.088 \\
\hline CRP (mg/L) & $12.57 \pm 5.95$ & $13.61 \pm 5.99$ & $14.11 \pm 6.18$ & 0.367 \\
\hline LVEF (\%) & $65.03 \pm 11.73$ & $63.13 \pm 10.43$ & $61.07 \pm 9.32$ & 0.216 \\
\hline Diabetes (\%) & $2(4.76 \%)$ & $6(12.24 \%)$ & $14(23.73 \%)$ & 0.025 \\
\hline Hypertension (\%) & $4(9.52 \%)$ & $6(12.24 \%)$ & $19(32.2 \%)$ & 0.005 \\
\hline
\end{tabular}

BMI: body mass index; TC: total cholesterol; TG: triglyceride; LDL-C: low-density lipoprotein cholesterol; HDL-C: high-density lipoprotein cholesterol; Hcy: homocysteine; CRP: C-reactive protein; LVEF: left ventricular ejection fraction.

3.4. Relationship between Levels of CCHE1 and TCF21 and Severity and Numbers of Vessel Lesions in CAD. Spearman's rank correlation results uncovered that CCHE1 level was positively correlated to CAD severity and numbers of vessel lesions ( $r=0.388$ and 0.671 , respectively, $p<0.05$ ), whereas TCF21 displayed a negative correlation to them $(r=-0.523$ and -0.397 , respectively, $p<0.05$ ) (Table 3 ). It is further supported that CCHE1 and TCF21 were involved in the progression of CAD. In particular, CCHE1 aggravated CAD and TCF21 exerted a protective role.

3.5. Negative Interaction between CCHE1 and TCF21 in $C A D$ Patients. Pearson's correlation test was conducted to elucidate the potential interaction between CCHE1 and TCF21 in CAD patients. As data revealed, the serum level of CCHE1 was negatively correlated to TCF21 $(r=-0.5099$, $p<0.001$ ) (Figure 3).

3.6. Influences of CCHE1 and TCF21 Levels on Cardiovascular Events in CAD Patients. During the 3-year follow-up, the incidence of cardiovascular events was $39.3 \%$ (59/150). Variables with statistical significances in Tables 1 and 2 were assigned as independent ones as follows: diabetes $\quad($ yes $=1$, no $=0)$; hypertension $\quad($ yes $=1$, no $=0)$; drinking (yes $=1$, no $=0$ ); CAD severity (mild lesion $=1$, moderate and severe lesion $=2$ ); and numbers of vessel 


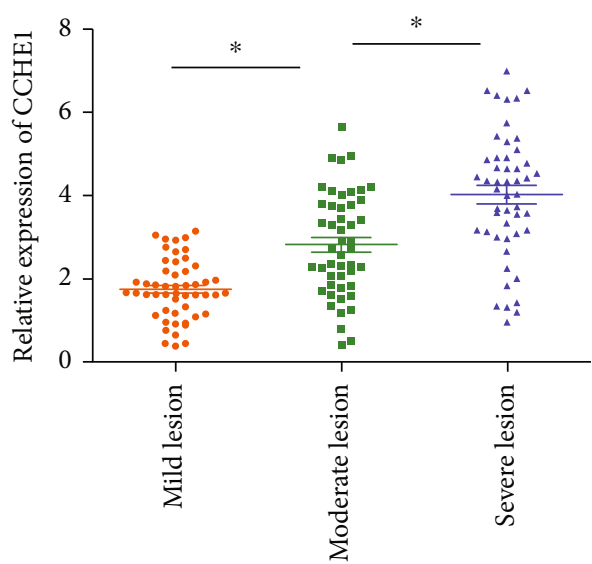

(a)

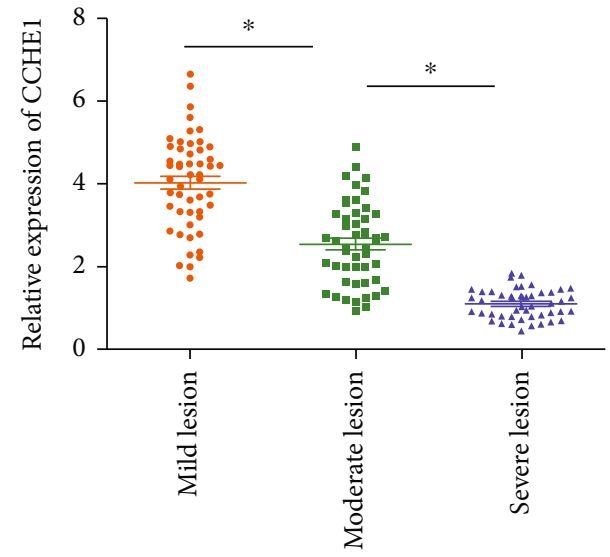

(b)

FIGURE 1: Serum levels of CCHE1 and TCF21 in CAD patients with different severities. Serum levels of CCHE1 (a) and TCF21 (b) in CAD patients of the mild lesion, moderate lesion, and severe lesion groups.

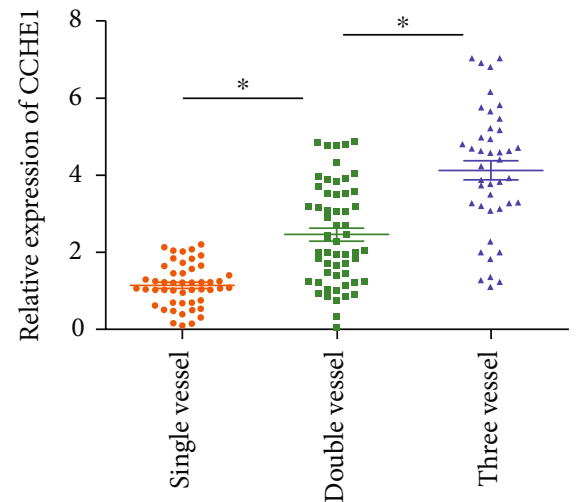

(a)

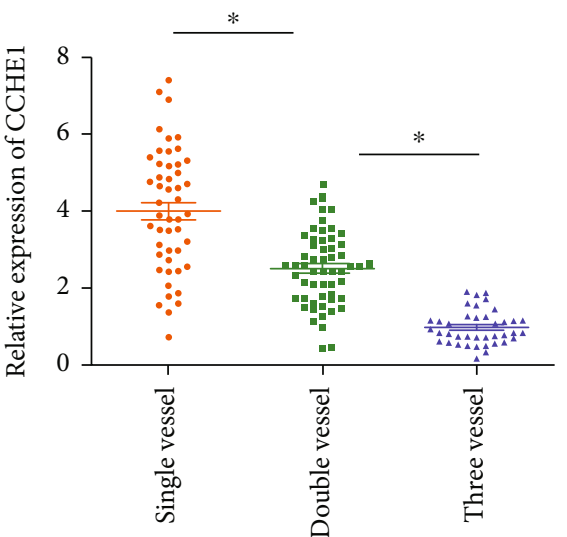

(b)

FIGURE 2: Serum levels of CCHE1 and TCF21 in CAD patients with different numbers of vessel lesions. Serum levels of CCHE1 (a) and TCF21 (b) in CAD patients of the single-vessel lesion, two-vessel lesion, and three-vessel lesion groups.

TABLE 3: Relationship between levels of CCHE1 and TCF21 and severity and numbers of vessel lesions in CAD.

\begin{tabular}{lcccc}
\hline & \multicolumn{2}{c}{ CCHE1 } & \multicolumn{2}{c}{ TCF21 } \\
& $r$ & $p$ & $r$ & $p$ \\
\hline Severity of CAD & 0.388 & 0.041 & -0.523 & 0.022 \\
Numbers of vessel lesions & 0.671 & 0.003 & -0.397 & $<0.001$ \\
\hline
\end{tabular}

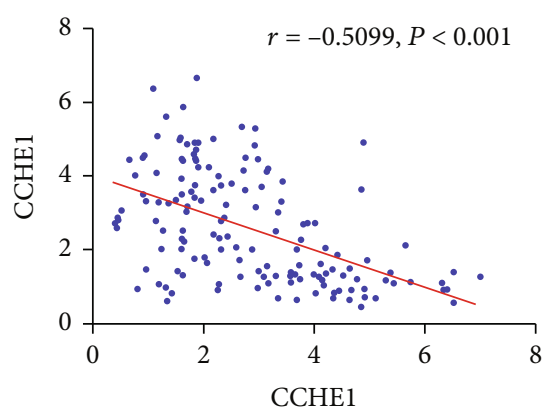

Figure 3: A negative correlation between serum levels of CCHE1 and TCF21 in CAD patients $(r=-0.5099, p<0.001)$.
TABLE 4: Multivariable logistic analysis on potential factors influencing the occurrence of cardiovascular events in CAD patients.

\begin{tabular}{lccc}
\hline Variable & OR & $95 \%$ CI & $p$ \\
\hline Diabetes & 1.733 & $0.614-5.332$ & 0.142 \\
Hypertension & 0.892 & $0.561-2.313$ & 0.668 \\
Drinking & 0.873 & $0.446-2.985$ & 0.079 \\
Severity of CAD & 1.681 & $1.281-3.452$ & 0.035 \\
Number of vessel lesions & 1.546 & $1.332-2.775$ & $<0.001$ \\
CCHE1 & 2.387 & $1.654-5.872$ & $<0.001$ \\
TCF21 & 0.584 & $0.287-1.664$ & 0.007 \\
\hline
\end{tabular}

lesions ( single vessel $=1$, multiple vessels $=2$ ). Subsequently, CAD patients were divided into two groups according to the cut-off values of mRNA levels of CCHE1 and TCF21 (high level $=1$, low level $=0$ ). The occurrence of cardiovascular events during the follow-up of CAD patients was assigned as the dependent variable (yes $=1$, no $=0$ ). A multivariable logistic regression test demonstrated that severe 
CAD, multiple vessel lesions, and high level of CCHE1 were independent risk factors, while the high level of TCF21 was a protective factor influencing the occurrence of cardiovascular events in CAD patients (Table 4).

\section{Discussion}

With the completion of the gene sequencing program and the development of molecular biology, it is found that protein-encoding mRNAs only account for $2 \%$ of the human genome. More than $95 \%$ of the transcribed sequences in the genome are noncoding RNAs [18]. These noncoding RNAs, which were initially regarded as nonfunctional, are involved in various life activities. As a family member of noncoding RNAs, lncRNAs are $200 \mathrm{bp}$ long. They exert diverse functions as signal molecules, decoy molecules, and scaffold molecules. IncRNAs are involved in genetic, transcriptional, and posttranscriptional regulations [19].

lncRNAs have been identified to have a relation to vascular diseases. It is reported that lncRNA ANRIL is highly expressed in the blood of atherosclerosis patients, and the high level of ANRIL enhances the risk of MI [20, 21]. Circulating system lncRNAs are promising biomarkers for diagnosing ischemic heart diseases. For example, lncRNA LIPCAR is linked to the progression of heart failure, which serves as a molecular indicator for myocardial remodeling [22]. Upregulated plasma lncRNA CoroMarker is highly specific and sensitive for diagnosing CAD [23]. Through regulating VSMC phenotypes, IncRNAs regulate atherosclerosis development by mediating adipogenesis and lipid deposition [24].

CCHE1 is upregulated in cervical cancer specimens and correlated to tumor size, tumor staging and overall survival in cervical cancer patients. It is considered a potential therapeutic target and prognostic biomarker for cervical cancer [25]. Meanwhile, CCHE1 is highly expressed in non-smallcell lung carcinoma, which drives malignant phenotypes via the ERK/MAPK signaling [26]. Overexpression of CCHE1 triggers proliferative rate and inhibits apoptosis of gastric cancer cells [27]. Our findings detected an increased serum level of CCHE1 in CAD patients, and its level was elevated with the aggravation of CAD. The high level of CCHE1 enhanced the risk of cardiovascular events in CAD patients.

TCF21, also known as POD-1, belongs to the bHLH family. It is expressed in the mesenchymal cells of the lung, kidney, and intestine; cardiac interstitial cells [28]; and glomerular epithelial cells [29]. TCF21 is of significance in driving EMT [30-32]. Cao et al. [31] detected urine samples in 50 bladder cancer patients, 50 renal cancer patients, and 50 prostate cancer patients. They proposed that combined detection of TCF21 level and PCDH17 methylation is conductive to diagnose urinary system tumors. Ye et al. [33] showed that abnormal methylation of TCF12 is associated with the poor prognosis in clear cell renal cell carcinoma. TCF21 is also an independent factor influencing the progression of CAD $[34,35]$. Consistently, we found that the serum level of TCF21 decreased in CAD patients. TCF21 exerted a protective role in $\mathrm{CAD}$ severity and numbers of vessel lesions. A negative correlation was identified between serum levels of CCHE1 and TCF21. We believed that enhanced level of CCHE1 and downregulated TCF21 predict the deterioration of CAD. In summary, we uncover the relationship between CCHE1 and TCF21 in CAD; however, the lack of in vivo and in vitro assay is the shortage of this research.

\section{Conclusion}

Increased serum level of CCHE1 and decreased TCF21 level are closely related to CAD severity, which are able to influence the prognosis in CAD patients.

\section{Data Availability}

The datasets used and analyzed during the current study are available from the corresponding author on reasonable request.

\section{Conflicts of Interest}

The authors declared no conflict of interest.

\section{Acknowledgments}

This study is supported by the Natural Science Foundation of Shaanxi Province (2020JQ-535).

\section{References}

[1] X. Dai, S. Wiernek, J. P. Evans, and M. S. Runge, "Genetics of coronary artery disease and myocardial infarction," World Journal of Cardiology, vol. 8, no. 1, pp. 1-23, 2016.

[2] X. B. Wang, Y. D. Han, S. Sabina et al., "HDAC9 variant Rs2107595 modifies susceptibility to coronary artery disease and the severity of coronary atherosclerosis in a Chinese Han population," PLoS One, vol. 11, no. 8, article e160449, 2016.

[3] S. Gao, M. Wassler, L. Zhang et al., "MicroRNA-133a regulates insulin-like growth factor-1 receptor expression and vascular smooth muscle cell proliferation in murine atherosclerosis," Atherosclerosis, vol. 232, no. 1, pp. 171-179, 2014.

[4] M. Sun and W. L. Kraus, "From discovery to function: the expanding roles of long noncoding RNAs in physiology and disease," Endocrine Reviews, vol. 36, no. 1, pp. 25-64, 2015.

[5] Y. Cai, Y. Yang, X. Chen et al., "Circulating "LncPPAR $\delta$ " from monocytes as a novel biomarker for coronary artery diseases," Medicine (Baltimore), vol. 95, no. 6, article e2360, 2016.

[6] J. Hou, H. Long, C. Zhou et al., "Long noncoding RNA Braveheart promotes cardiogenic differentiation of mesenchymal stem cells in vitro," Stem Cell Research \& Therapy, vol. 8, no. 1, p. 4, 2017.

[7] Z. Zhang, W. Gao, Q. Q. Long et al., "Increased plasma levels of lncRNA H19 and LIPCAR are associated with increased risk of coronary artery disease in a Chinese population," Scientific Reports, vol. 7, no. 1, p. 7491, 2017.

[8] F. Fasolo, H. Jin, G. Winski et al., "Long noncoding RNAMIATControls advanced atherosclerotic lesion formation and plaque destabilization," Circulation, vol. 144, no. 19, pp. 1567-1583, 2021.

[9] M. Li, H. Zheng, Y. Han et al., "LncRNA Snhg1-driven selfreinforcing regulatory network promoted cardiac regeneration 
and repair after myocardial infarction," Theranostics, vol. 11, no. 19, pp. 9397-9414, 2021.

[10] M. Yang, X. Zhai, B. Xia, Y. Wang, and G. Lou, "Long noncoding RNA CCHE1 promotes cervical cancer cell proliferation via upregulating PCNA," Tumour Biology, vol. 36, no. 10, pp. 7615-7622, 2015.

[11] Y. Chen, C. X. Wang, X. X. Sun, C. Wang, T. F. Liu, and D. J. Wang, "Long non-coding RNA CCHE1 overexpression predicts a poor prognosis for cervical cancer," European Review for Medical and Pharmacological Sciences, vol. 21, no. 3, pp. 479-483, 2017.

[12] W. Peng and H. Fan, "Long noncoding RNA CCHE1 indicates a poor prognosis of hepatocellular carcinoma and promotes carcinogenesis via activation of the ERK/MAPK pathway," Biomedicine \& Pharmacotherapy, vol. 83, pp. 450-455, 2016.

[13] L. T. Smith, M. Lin, R. M. Brena et al., "Epigenetic regulation of the tumor suppressor gene TCF21 on 6q23-q24 in lung and head and neck cancer," Proceedings of the National Academy of Sciences of the United States of America, vol. 103, no. 4, pp. 982-987, 2006.

[14] X. Ao, W. Ding, Y. Zhang, D. Ding, and Y. Liu, “TCF21: a critical transcription factor in health and cancer," Journal of Molecular Medicine, vol. 98, no. 8, pp. 1055-1068, 2020.

[15] X. Guo, Y. Wei, Z. Wang et al., "LncRNA LINC00163 upregulation suppresses lung cancer development though transcriptionally increasing TCF21 expression," American Journal of Cancer Research, vol. 8, no. 12, pp. 2494-2506, 2018.

[16] H. Jneid, J. L. Anderson, R. S. Wright et al., "2012 ACCF/AHA focused update of the guideline for the management of patients with unstable angina/non-ST-elevation myocardial infarction (updating the 2007 guideline and replacing the 2011 focused update): a report of the American College of Cardiology Foundation/American Heart Association Task Force on Practice Guidelines," Journal of the American College of Cardiology, vol. 60, no. 7, pp. 645-681, 2012.

[17] G. G. Gensini, "A more meaningful scoring system for determining the severity of coronary heart disease," The American Journal of Cardiology, vol. 51, no. 3, p. 606, 1983.

[18] E. Pennisi, "Shining a light on the genome's 'dark matter," Science, vol. 330, no. 6011, p. 1614, 2010.

[19] J. T. Kung, D. Colognori, and J. T. Lee, "Long noncoding RNAs: past, present, and future," Genetics, vol. 193, no. 3, pp. 651-669, 2013.

[20] A. Helgadottir, G. Thorleifsson, A. Manolescu et al., "A common variant on chromosome 9p21 affects the risk of myocardial infarction," Science, vol. 316, no. 5830, pp. 1491-1493, 2007.

[21] R. Murray, J. Bryant, P. Titcombe et al., "DNA methylation at birth within the promoter of ANRIL predicts markers of cardiovascular risk at 9 years," Clinical Epigenetics, vol. 8, no. 1, p. 90, 2016.

[22] R. Kumarswamy, C. Bauters, I. Volkmann et al., "Circulating long noncoding RNA, LIPCAR, predicts survival in patients with heart failure," Circulation Research, vol. 114, no. 10, pp. 1569-1575, 2014.

[23] Y. Yang, Y. Cai, G. Wu et al., "Plasma long non-coding RNA, CoroMarker, a novel biomarker for diagnosis of coronary artery disease," Clinical Science (London, England), vol. 129, no. 8, pp. 675-685, 2015.
[24] S. Greco, G. Zaccagnini, A. Perfetti et al., "Long noncoding RNA dysregulation in ischemic heart failure," Journal of Translational Medicine, vol. 14, no. 1, p. 183, 2016.

[25] N. Naghashi and S. Ghorbian, "Clinical important dysregulation of long non-coding RNA CCHE1 and HULC in carcinogenesis of cervical cancer," Molecular Biology Reports, vol. 46, no. 5, pp. 5419-5424, 2019.

[26] Y. Liao, S. Cheng, J. Xiang, and C. Luo, "IncRNA CCHE1 increased proliferation, metastasis and invasion of non-small lung cancer cells and predicted poor survival in non-small lung cancer patients," European Review for Medical and Pharmacological Sciences, vol. 22, no. 6, pp. 1686-1692, 2018.

[27] G. Xu, Y. Zhang, N. Li, J. B. Zhang, and R. Xu, "LncRNA CCHE1 in the proliferation and apoptosis of gastric cancer cells," European Review for Medical and Pharmacological Sciences, vol. 22, no. 9, pp. 2631-2637, 2018.

[28] S. E. Quaggin, L. Schwartz, S. Cui et al., "The basic-helix-loophelix protein pod1 is critically important for kidney and lung organogenesis," Development, vol. 126, no. 24, pp. 57715783, 1999.

[29] S. E. Quaggin, G. B. vanden Heuvel, and P. Igarashi, "Pod-1, a mesoderm-specific basic-helix-loop-helix protein expressed in mesenchymal and glomerular epithelial cells in the developing kidney," Mechanisms of Development, vol. 71, no. 1-2, pp. 3748, 1998.

[30] B. Baum, J. Settleman, and M. P. Quinlan, "Transitions between epithelial and mesenchymal states in development and disease," Seminars in Cell \& Developmental Biology, vol. 19, no. 3, pp. 294-308, 2008.

[31] H. Cao, E. Xu, H. Liu, L. Wan, and M. Lai, "Epithelial-mesenchymal transition in colorectal cancer metastasis: a system review," Pathology, Research and Practice, vol. 211, no. 8, pp. 557-569, 2015.

[32] A. Mitra, L. Mishra, and S. Li, "EMT, CTCs and CSCs in tumor relapse and drug-resistance," Oncotarget, vol. 6, no. 13, pp. 10697-10711, 2015.

[33] Y. W. Ye, Z. M. Jiang, W. H. Li et al., "Down-regulation of TCF21 is associated with poor survival in clear cell renal cell carcinoma," Neoplasma, vol. 59, no. 6, pp. 599-605, 2012.

[34] C. L. Miller, D. R. Anderson, R. K. Kundu et al., "Diseaserelated growth factor and embryonic signaling pathways modulate an enhancer of TCF21 expression at the 6q23.2 coronary heart disease locus," PLoS Genetics, vol. 9, no. 7, article e1003652, 2013.

[35] V. L. Costa, R. Henrique, S. A. Danielsen et al., "TCF21 and PCDH17 methylation: an innovative panel of biomarkers for a simultaneous detection of urological cancers," EpigeneticsUs, vol. 6, no. 9, pp. 1120-1130, 2011. 